\author{
SUMMARY \\ INTEREST OF USING A COLD MUTANT REARED IN CELLULAR CULTURE \\ FOR THE VACCINATION AGAINST USUAL SWINE FEVER
}

Non pathogenic mutants of the swine fever virus were isolated by means of immuno-fluorescence techniques after selection in cellular culture at $29-30^{\circ}$. These mutants may be easily differentiated from the virulent savage strains by means of $i n$ vitro determination of the genetic markers related with the pathogenic capacity :

The virulent savage virus is hot (optimum temperature at $4^{\circ}{ }^{\circ} \mathrm{C}$ ) and solid (resistant at $56^{\circ} \mathrm{C}$ ) whereas the non pathogenic mutant isolate at $29-30^{\circ} \mathrm{C}$ is cold (optimum temperature at $30-33^{\circ} \mathrm{C}$ ) and fragile (inactivated at $56^{\circ} \mathrm{C}$ ).

The mutants have no residual pathogenic capacity and when they are inoculated into piglets treated or not with immuno-depressors, they are not able to provoke hyperthermia or clinical symptomes.

Because of the immunity obtained by means of these mutants, the animals resist to the test inoculation with the savage virus.

Offering a guaranty of innocuousness and efficiency, these virus may be used as alive vaccines without hyperimmune homologous serum, which is a great economic and practical advantage.

\title{
IMPORTANCE EN FRANCE DE LA COLIBACILLOSE DU PORCELET NOUVEAU-Né
}

\author{
L. RENAULT, C. MAIRE et J. VAISSAIRE \\ Établissements Sanders, 91 - Juvisy-sur-Orge
}

\section{RÉSUMÉ}

La recherche systématique des souches pathogènes d'Escherichia coli, par la mise en évidence de leur pouvoir hémolytique, de leur pouvoir agglutinogène direct en présence de sérums anticap. sulaires et de leur pouvoir pathogène pour la souris, permet de constater que la colibacillose est une des principales affections digestives, sinon la principale, du porcelet nouveau-né. Sur 235 élevages présentant des troubles, $46,8 \mathrm{p}$. Ioo d'entre eux étaient atteints de cette maladie pour une période allant de 1966 aux trois premiers trimestres $197^{\circ}$.

Un examen bactériologique précis doit permettre la mise en place d'un traitement antibio.tique efficace ou d'un vaccin approprié à l'élevage, dans la mesure où il portera sur un grand nombre de porcelets et de matières fécales des truies gestantes. Ces mesures ne pourront bien entendu remplacer, dans aucun cas, les mesures classiques d'hygiène concernant les animaux et les bâtiments. 


\title{
SUMMARY
}

\section{IMPORTANCE IN FRANCE OF COI,IBACILIOSIS IN THE NEWBORN PIG}

Systematic research of pathological strains of E. Coli by the means of an hemolytic test, a rapid slide agglutination test using $E$. coli $O K$ serum and by virulence test for mice, suggests that colibacillosis is one of the main digestive troubles, if not the most important, in the newborn pig.

Out of 235 farms having troubles, 46.8 p. Ioo were affected with this disease from I 966 to the first three terms of 1970 .

By means of a precise bacteriological examination it should be possible to apply an efficient antibiotic treatment or a farm adapted vaccine. This examination must be carried out on a large number of piglets and feces of pregnant sows.

However, these methods will never replace good sanitation practices concerning animals and their housing.

\section{ULCÈRE GASTRO-GSOPHAGIEN DU PORC ; ESSAIS DE PROPHYLAXIE}

\author{
J. TOURNUT et C. LABIE
}

Chaire d'Hygiène et Industrie des Aliments d'Origine animale, Chaire de Pathologie médicale du Bétail et des Animaux de Basse-cour, École nationale vétérinaire de Toulouse I. N. R. A.,

Chemin des Capelles, 31 - Toulouse

\section{RÉSUMÉ}

Ayant émis l'hypothèse que les ulcères gastro-œsophagiens étaient la conséquence d'un trouble de la sécrétion gastrique et que les facteurs ulcérogènes pouvaient intervenir sur une ou plusieurs phases de cette dernière, les auteurs ont établi un protocole expérimental pour tenter de le démontrer.

Le facteur ulcérogène choisi a été la finesse de la mouture.

En utilisant le Méprobamate (action sur le SN central) et l'Ester Sulfate de Galactopyranose (action gastrique), les auteurs obtiennent des résultats encourageants.

L'association des 2 produits (300 $\mathrm{mg}$ pour le Méprobamate et $2 \mathrm{~g}$ d'Ester Sulfate de Galactopyranose par kg d'aliment) diminue considérablement les lésions d'hyperkératose et agit favorablement sur la croissance. 